\title{
A Study on a Drawing Tool of a Spatial Drawing Application in Virtual Reality
}

\author{
Ginam $\mathrm{Ko}^{1}$, Kyoo-Won Suh ${ }^{2}$, SangHun $\mathrm{Nam}^{3} *$ \\ ${ }^{1}$ placeB Inc, Guro-gu, Seoul, South Korea \\ ${ }^{2}$ Industry Academic Cooperation Foundation, Hallym University, Chuncheon-Si, \\ Gangwon-Do, South Korea \\ 3* Media Businsss, Dept. Of Newmedia, Seoul Media Institute of Technology, 99, \\ Hwagok-ro 61-gil, Gangseo-gu, Seoul, South Korea \\ 1'gnko@placeb.com, ${ }^{2}$ suh7164@hallym.ac.kr, ${ }^{3}$ shnam@smit.ac.kr
}

\begin{abstract}
A system for spatial drawing must have a display device that shows stereoscopic images to the user, a controller serving as a brush for drawing, and a function that creates a line and a curved surface and visualizes the virtual space. Herein, a software framework was designed for spatial drawing by analyzing the functions for spatial drawing applications in a virtual environment based on existing studies and produced a virtual reality spatial drawing application with virtual drawing tools. The application employed a traditional painting metaphor. Furthermore, a brush module and a palette module were designed for the spatial drawing interface and linked to the line and surface generator modules to change drawing attributes, e.g., color and texture.
\end{abstract}

Keywords: Virtual Reality, Spatial Drawing, Drawing Tool. ${ }^{1}$

\section{Introduction}

For a long period time, humans have used images for sharing information and delivering messages. They have experienced the joy of creation through aesthetic drawings. Drawings on the walls of caves, ancient Egyptian hieroglyphics, and fresco murals in the Renaissance have aimed to tell stories using images, and in the modern era, drawing is a familiar method of expression that presents visual and tactile experiences to humans, e.g., sketching on a paper with a pencil or coloring using paints [1]. With the advancement of digital technology and the distribution of computers, many people have started using keyboards and mouses, the two most representative user interface devices that have created an interface gap between traditional drawing interfaces used by designers and artists. However recently, many smart devices and computers have started using pen-type interface devices and smartphones implemented with touch interfaces. These advancements have made it possible to interact with intuitive interfaces using hands again. In particular, virtual reality (VR) technologies visualize three-dimensional (3D) images in real time to users by using immersive display devices, e.g., cave automatic virtual environment (CAVE) and head-mounted displays (HMDs) to expand the scope of expression from the flat surface to a space and change the paradigm of drawing.

Article history:

Received (May 16, 2018), Review Result (June 15, 2018), Accepted (July 17, 2018) 
'Cave Painting' showcased a study that draws images in a space using an actual brush-like device in the CAVE system 2]. Tilt Brush and Quill are representative VR painting applications with which the user can wear an HMD and engage in a creative painting process freely in a 3D space [3][4]. The act of drawing in a space has advanced from exhibitions to the performance of drawing in front of many audiences. Furthermore, educational courses for spatial drawing have emerged. As the needs of the development of spatial drawing applications based on VR technologies increase, this study focus on virtual drawing tools based on the spatial drawing framework via analysis of the existing spatial drawing studies. We implemented the VR spatial drawing application and proposed virtual drawing tools for the application.

\section{Spatial Drawing Framework Design}

A spatial drawing system comprises a display device that shows stereoscopic images, a controller that serves as a brush and palette for drawing, and a software package that creates lines and curved surfaces in a virtual space. As shown in Figure 1, the spatial drawing framework structure is designed based on previous studies on several spatial drawing applications. In the spatial drawing process, a user draws a line and a surface using a handtracking device. The hand-tracking module acquires the position and orientation data from HMD controllers and the hand joints or fingertips of the hand-tracking device, e.g., Leap Motion [5]. The head-tracking module tracks the movement of the HMD and the 3D visualization module updates the virtual environment in real time based on the head-tracking module [6].

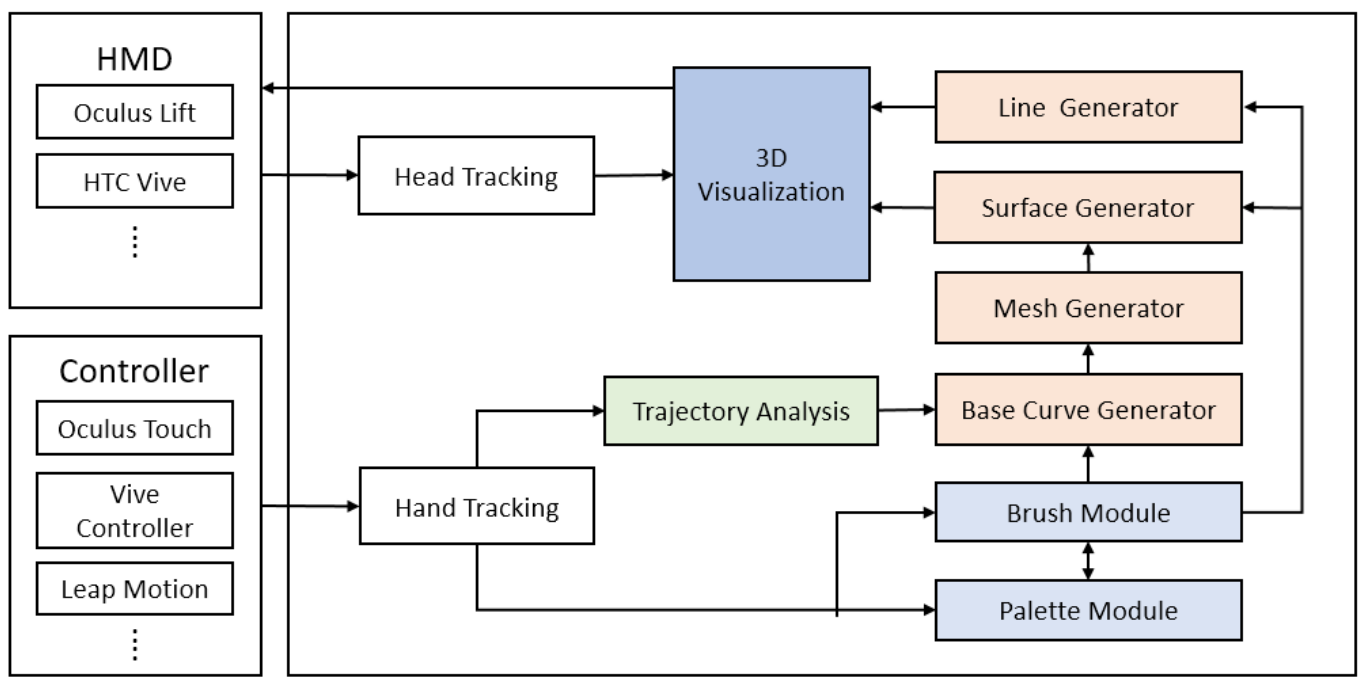

Figure 1. Structure of the spatial drawing framework

The trajectory analysis module calculates the curvature and velocity from continuous input data obtained from the hand-tracking module. The base curve generator creates base curves that show the center of the curved surface using the position data obtained from the trajectory analysis module. The base curve generator generates efficient base curves using the velocity and curvature obtained from the trajectory analysis module. The mesh generator creates curved meshes using the base curve and the orientation of the controller. The user can set drawing attributes such as the color and texture on the curved surface using virtual drawing tools, e.g., a brush and a palette. The line generator creates lines with the color of the brush module. The 
surface generator creates various types of curved surfaces by implementing the color or texture set on the brush module.

\section{Virtual Drawing Tools of the VR Spatial Drawing System}

Spatial drawing applications support virtual drawing tools to create various lines and curved surfaces. Users can use the brush and palette menus to set the attributes of the line and surface color and texture in a virtual environment. The brush menu can be manipulated by the user with a controller, and these settings are saved in the spatial drawing application when lines and curved surfaces need to be generated using the line generator and the surface generator. As shown in Figure 2, virtual drawing tools in spatial drawing applications can be designed diversely [7]. Since the spatial drawing tool, which is different from the tools used for a flat monitor, is placed in the virtual environment, the brush and palette menus must be modeled in 3D. Depending on the number of HMD controllers or the number of hands, the virtual drawing tools must design a brush menu and a palette menu design suitable for single-handed and twohanded interfaces.

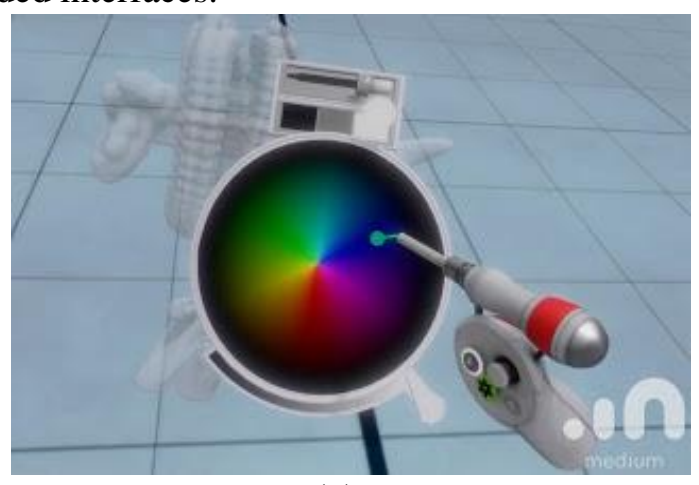

(a)

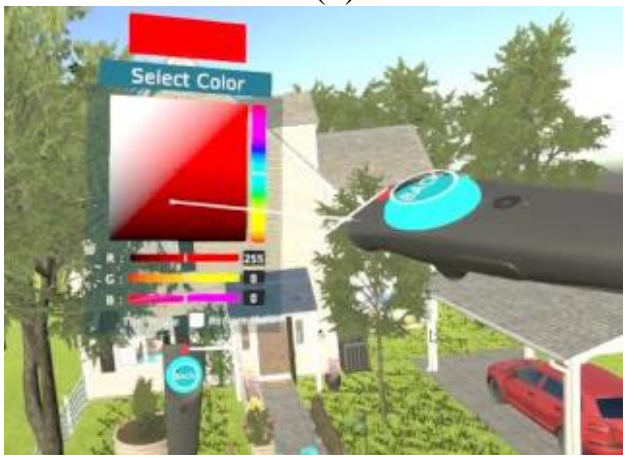

(c)

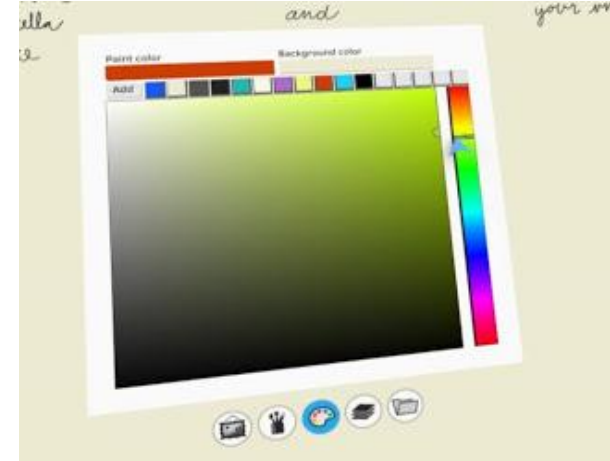

(b)

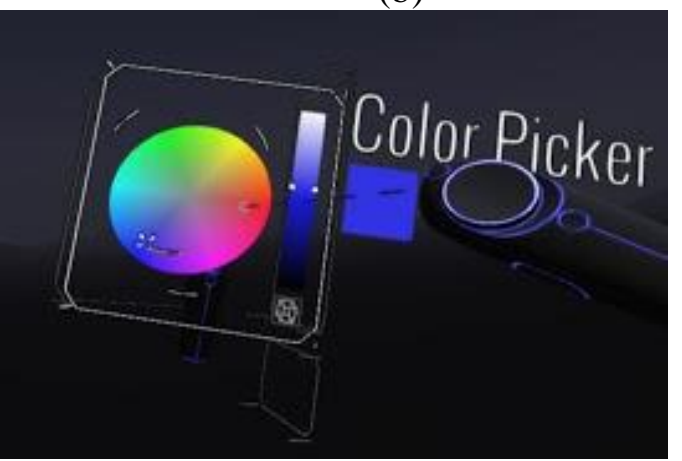

(d)

Figure 2. The design of virtual drawing tools in virtual reality: (a) Medium by Oculus, (b) Quill by Story Studio, (c) Modbox by Alientrap, and (d) Tile Brush by Google.

The spatial drawing application is implemented based on the spatial drawing framework. The virtual drawing tool of the proposed application uses the metaphor of traditional painting. The tool supports a two-hand interface. The brush module is connected to the dominant hand, and the palette module is connected to the non-dominant hand. The brush module is required to determine the attributes for lines and surfaces and visualize the selected attributes to the user. In the line-drawing mode, the brush module is expressed in the shape of a sphere as shown in 
Figure 3. The size of the sphere represents the thickness of the line drawn. The user can change the radius of the sphere using the touch pad of the controller. If the sphere makes contact with the palette module, the color of the palette that made contact with the sphere is used as the color of the sphere. In surface-drawing mode, the brush module is expressed in the shape of four prisms as shown in Figure 3. The length of the shape represents the width of a surface drawn. Using the touch pad of the controller, the length of the four prisms can be changed. If the shape makes contact with the palette, the color of the palette is used as the drawing color of the curved surface.
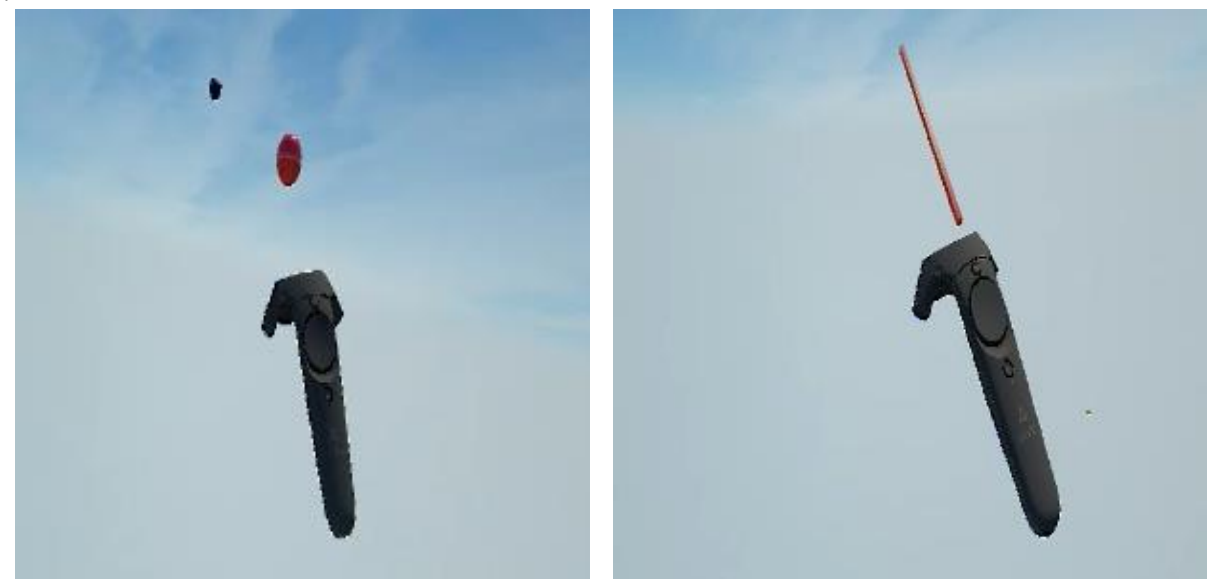

Figure 3. Brush module for drawing a line and a surface

The palette module is designed as a circular plate with color blocks. In this study, this module expresses key colors and the developer of the drawing application sets the RGB values of the color blocks. These blocks have an area for detecting collision with the brush module. When the collision occurs, the palette module sends the color data to the brush module based on the selected color block.
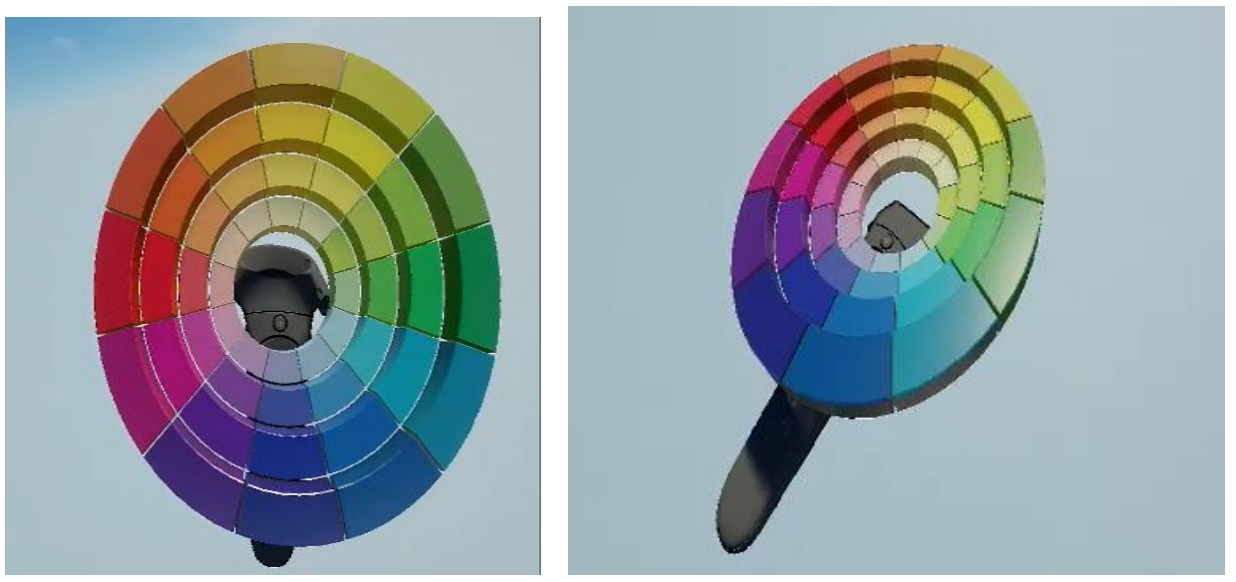

Figure 4. Palette module with color blocks 


\section{Conclusion}

Our research analyzed existing spatial drawing studies conducted in VR environment. This topic has gained increasing attention in arts and entertainment industry. We designed a drawing framework that could be used for creating spatial drawing applications. Based on the spatial drawing framework, a VR-based spatial drawing application was developed with the aid of Coexistent Reality Software Framework engine and HTC Vive as hardware [8]. In particular, this study investigates a virtual drawing tool of a spatial drawing application and implements the drawing tool with the brush and palette modules. The virtual drawing module has the metaphor of traditional painting for an intuitive user interface. The controller of the dominant hand connects to the brush module that displays different shapes according to the drawing mode. The controller of non-dominant hand connects to the palette module that displays a circular plate with color blocks. The line and surface generators allow the user to set the color and texture using the drawing tool in a virtual space.

\section{Acknowledgements}

This research was supported by Basic Science Research Program through the National Research Foundation of Korea (NRF) funded by the Ministry of Education (No. NRF2017R1D1A1B03034041)

\section{References}

[1] C. Tricart. Virtual Reality Filmmaking: Techniques \& Best Practices for VR Filmmakers. Taylor \& Francis. (2017)

[2] D. F. Keefe, D. A. Feliz, T. Moscovich, D. H. Laidlaw and J. J. LaViola Jr.. CavePainting: a fully immersive 3D artistic medium and interactive experience. Proceedings of the 2001 symposium on Interactive 3D graphics, (2001) March 19-21; North Carolina, USA.

[3] Tilt Brush, https://www.tiltbrush.com (2018), Accessed: 2018-06- 01.

[4] Quill, https://www.oculus.com/experiences/rift/1118609381580656/ (2018), Accessed: 2018-06- 01.

[5] C. M. Wu, C. W. Hsu, T. K. Lee and S. Smith. A virtual reality keyboard with realistic haptic feedback in a fully immersive virtual environment. Virtual Reality. 21, (2017)

[6] D. Bowman, E. Kruijff, J. J. LaViola Jr and I. P. Poupyrev. 3D User interfaces: theory and practice. AddisonWesley, Boston, (2004)

[7] Tool Pallet design in virtual reality, https://www.etc.cmu.edu/projects/blueprint/index.php/2017/02/22/toolpallet-design-in-virtual-reality/ (2018), Accessed: 2018-06-01.

[8] CRseed Studio, http://www.crseed.org/ (2018), Accessed: 2018-06- 01.

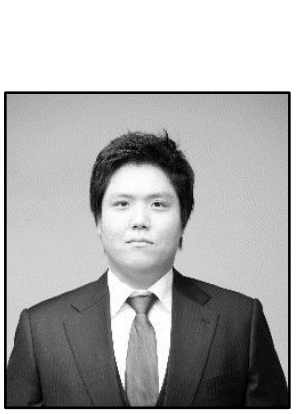

\section{Authors}

\section{Ginam Ko}

He received the M.S degrees in Convergence Engineering from Hoseo Graduate School of Venture, Seoul, South Korea in 2013, and he has completed the Ph.D coursework in Convergence Engineering in Hoseo Graduate School of Venture, Seoul, South Korea in 2016.. He was the Research Engineer and Assistant Consultant at the Yong Consulting Inc., and $\mathrm{He}$ is founder and CEO of placeB Inc. and he is designing AR, VR services on New Media service area as a Chief Director. His research interests include New Media service, Augmented and Virtual Reality and Mixed Reality technologies, User Interface, and Haptics. 


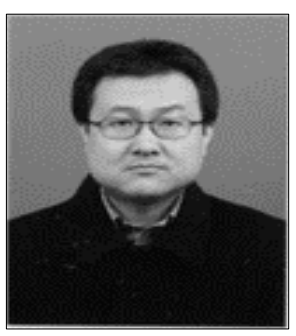

\section{Kyoo-Won Suh}

He received an MS Degree in business engineering from Korea Advanced Institute of S\&T (KAIST; Seoul, South Korea) in 2002 and completed a doctoral course. He was a senior researcher at the Korea Evaluation Institute of Industrial Technology (KEIT) and PM at the Center of Human-centered Interaction for Coexistence organized by the Korean government. Since 2017, he has been an associate professor at Industry Academic Cooperation Foundation, Hallym University, Chuncheon-Si, Gangwon-Do. His research interests include interactive media art and augmented reality ( $\mathrm{AR}$ )/virtual reality (VR)/mixed reality (MR) content for education, Internet of Medical Things (IOMT), R\&D planning and management, technology transfer and commercialization, and startups.

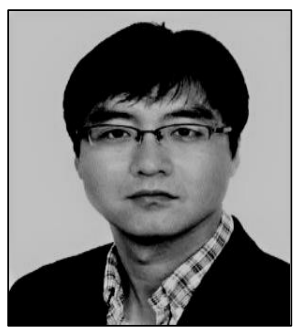

\section{SangHun Nam}

He received a BS degree in mechanical design from the Chung-Ang University, Seoul, South Korea. Further, he received an MS degree in computer graphics and virtual environment from Graduate School of Advanced Imaging Science, Multimedia and Film, Chung-Ang University, in 2001. In addition, he received a $\mathrm{PhD}$ degree in 2012. $\mathrm{He}$ was a senior researcher at the Center of Human-Centered Interaction for Coexistence organized by the Korean government. Since 2017, he has been an assistant professor at New Media, Seoul Media Institute of Technology. His research interests include user interface and experience, games, and biomedical technology in VR and AR, the design and expression of digital human in coexistent reality, and interactive media art for children education. 